\title{
Hydroxybutyrate promotes the recovery from cerebral infarction by activating Amp-activated protein kinase signaling
}

\author{
HUISHENG WU, PEIPEI GUO, XINYI LI, ZHAO JIN, XIN YANG and YANLIN WANG \\ Department of Anesthesiology, Zhongnan Hospital of Wuhan University, Wuhan, Hubei 430071, P.R. China
}

Received December 19, 2017; Accepted June 6, 2018

DOI: $10.3892 /$ etm.2018.6304

\begin{abstract}
Recent studies have shown that hydroxybutyrate (GHB) is effective for protection against ischemia/brain damage in rat models. However, the specific underlying mechanism is poorly understood. In line with the previous studies, the present data showed that GHB improves cerebral blood flow $(\mathrm{CBF})$ and physiological variables, including $\mathrm{pH}$, $\mathrm{pCO}_{2}$ and $\mathrm{pO}_{2}$. Using CD31-immunofluorescence staining, a reduction of blood vessel density was indicated in the middle cerebral artery occlusion (MCAO) group; however, GHB treatment enhanced the cerebral vascular density in the ischemic area. In addition, GHB treatment increased the number of BrdU/lectin double-positive cells. Furthermore, the reduction of nestin-positive cells was identified in the brain of MCAO rats, while the number of nestin-positive cells was significantly increased after GHB administration. Compared with the sham group, the activation of Amp-activated protein kinase (AMPK) was identified in MCAO rats, suggesting stress-mediated AMPK activation after ischemia. Furthermore, the western blot assay showed that GHB treatment resulted in further activation of AMPK and endothelial nitric oxide synthase (eNOS), suggesting an enhanced energy supply. In summary, the present novel data indicates that GHB promotes the recovery from cerebral infarction mainly by activating AMPK and eNOS signaling, thereby enhancing angiogenesis and neuron regeneration.
\end{abstract}

\section{Introduction}

Stroke is a serious threat to human health around the world and is characterized by high incidence, high morbidity and high mortality $(1,2)$. At present, the cure for ischemic stroke is limited (3-5). For instance, recombinant tissue plasminogen activator (rtPA), which is approved by the Food and Drugs

Correspondence to: Dr Yanlin Wang, Department of Anesthesiology, Zhongnan Hospital of Wuhan University, 169 Donghu Road, Wuhan, Hubei 430071, P.R. China

E-mail: wuhuisheng003@126.com

Key words: hydroxybutyrate, cerebral infarction, Amp-activated protein kinase, eNOS
Administration (FDA), is effective only if its administration is performed within $4.5 \mathrm{~h}$ after the stroke (6). Due to the narrow therapeutic window, few patients benefit from it (6). Hence, it is important to develop novel drugs for ischemic stroke therapy.

As an important serine/threonine protein kinase, AMP-activated protein kinase (AMPK) is responsible for the peripheral energy balance $(7,8)$. When cellular energy supply is low, AMPK is activated, thereby enhancing the energy production (9). In the periphery, AMPK acutely modulates the homeostasis of cellular metabolism by reducing energy storage and increasing energy utilization. Furthermore, a high expression level of AMPK is observed in neurons, and it is quickly activated in the brain upon energy deprivation (10). In addition, endothelial nitric oxide synthase (eNOS), phosphorylation is enhanced by AMPK and NO production is hence increased, which then leads to the regeneration of vessels (11-13). Therefore, modulation of AMPK activity is an important intervention method for the treatment of cerebral infarction.

Hydroxybutyrate (GHB), derived from $\gamma$-aminobutyric acid, is a well-known neurotransmitter and neuromodulator interacting with the GHB receptor and the $\gamma$-aminobutyric acid type B receptor in the central nervous system (14). Generally, GHB is used by clinicians to treat cataplexy, excessive daytime sleepiness and sleep disturbance associated with narcolepsy $(15,16)$. Recent studies have shown that GHB is effective for protection against ischemia/brain damage in rat models $(17,18)$. However, the specific underlying mechanism is poorly understood.

In the current study, we mainly explored the GHB effect on ischemic stroke in rats. Here, we demonstrated, for the first time, that GHB enhanced the activation of AMPK and eNOS, thereby improving recovery from cerebral infarction.

\section{Materials and methods}

Drug administration in middle cerebral artery occlusion (MCAO) in rats. A total of 60 male Sprague Dawley (SD) rats weighing 200-240 g were used in this study. Rats were housed in the same animal care facility during a 12-h light/dark cycle throughout the duration of the protocol, with free access to food and water. Briefly, the rats were anesthetized via $2 \%$ pentobarbital sodium $(40 \mathrm{mg} / \mathrm{kg}$ ) injected i.p. (19). The rats were considered to be properly anesthetized once they were without pain reflex. Arterial blood samples obtained via a 
femoral catheter were collected to measure $\mathrm{pO}_{2}, \mathrm{pCO}_{2}$ and $\mathrm{pH}$ with an AVL 998 Blood Gas Analyzer (Roche Diagnostics, Basel, Switzerland). The rectal temperature was maintained at $37 \pm 0.5^{\circ} \mathrm{C}$ during $\mathrm{MCAO}$ via a temperature-regulated heating lamp. A fiber-optic probe was attached to the parietal bone overlaying the middle cerebral artery territory $5 \mathrm{~mm}$ posterior and $5 \mathrm{~mm}$ lateral to the bregma, and it was connected to a laser Doppler flowmeter (PeriFlux System 5000; Perimed, Stockholm, Sweden) for continuous monitoring of the cerebral blood flow (CBF). A 4-0 nylon monofilament suture with a heat-blunted tip was introduced into the internal carotid artery through the artery stump. It was gently advanced for a distance of $18 \mathrm{~mm}$ from the common carotid artery bifurcation to block the origin of the middle cerebral artery for $90 \mathrm{~min}$ and then withdrawn to allow reperfusion. After the wound had been closed, the animals were allowed to recover from anesthesia before they were returned to their home cages. Sixty adult male SD rats were randomly divided into a sham operation group, an ischemia-reperfusion group (MCAO) and an $\mathrm{MCAO}+\mathrm{GHB}$ treated group. The rats were intraperitoneally treated with either $50 \mu \mathrm{l}$ of saline or $100 \mathrm{mg} / \mathrm{kg} \mathrm{GHB}$ (Lipomed AG, Arlesheim, Switzerland) each day for 5 weeks starting from $24 \mathrm{~h}$ following the MCAO procedure.

All experimental protocols described in this study were approved by the Ethics Review Committee for Animal Experimentation of Zhongnan Hospital of Wuhan University Hospital (no. XAERC-1002).

Assessment of neurological deficit score and analysis of survival rates. For sham group ( $\mathrm{n}=4$ rats) and MCAO group $(n=4$ rats), the neurological deficit score was evaluated after $24 \mathrm{~h}$ of operation before the rats were sacrificed. For $\mathrm{MCAO}+\mathrm{GHB}$ treated group, the neurological deficit score was evaluated after the rats received GHB therapy ( $n=4$ rats) for 5 weeks before the rats were sacrificed. Then, the neurological deficit score was evaluated. Two examiners were blinded to the rat identities and the treatment protocol. The following neurological deficit scoring (NDS) system (20) was used: 0 , no motor deficits (normal); 1, forelimb weakness and torso turning to the ipsilateral side when held by the tail (mild); 2 , circling to the contralateral side but normal posture at rest (moderate); 3, unable to bear weight on the affected side at rest (severe); and 4, no spontaneous locomotor activity or barrel rolling (critical). If no deficit was observed $2 \mathrm{~h}$ post-recovery from anesthesia, the animal was removed from further study.

Edema measurement. The ipsilateral and contralateral hemispheres were dissected, and the wet weight of the tissue was determined. The tissues were dried at $120^{\circ} \mathrm{C}$ for $24 \mathrm{~h}$. The percent cerebral water was determined as (wet weight-dry weight)/dry weight x 100 .

Infarct analysis. At $72 \mathrm{~h}$ after stroke, the brain was removed and cut into 5 2-mm slices and stained with 1.5\% 2,3,5-triphenyltetrazolium (TTC) for $30 \mathrm{~min}$ at $3^{\circ} \mathrm{C}$. For sham group, $\mathrm{MCAO}$ group and $\mathrm{MCAO}+\mathrm{GHB}$ treated group $(\mathrm{n}=4$ rats for each group), the rats were anesthetized after the rats received saline or GHB therapy for 5 weeks via $2 \%$ pentobarbital sodium (40 mg/kg) injected i.p. (19). The rats were considered to be properly anesthetized once they were without pain reflex.
Then, they were perfused transcardially with cold PBS to assess chronically post-stroke, followed by $4 \%$ paraformaldehyde; the brain was post-fixed for $18 \mathrm{~h}$ and placed in cryoprotectant $(30 \%$ sucrose). The brain tissue was cut into $40-\mu \mathrm{m}$ free-floating sections on a freezing microtome and every eighth slice was stained by cresyl violet stain for evaluation of ischemic cell damage. Infarct volume, expressed as a percentage of whole-brain volume, was measured by an image-processing and analysis system (1.25xobjective, Q570IW; Leica, Wetzlar, Germany) and calculated by integration of the infarct area in each brain section along the rostral-caudal axis.

Western blot analysis. Western blots were performed as described previously (18). For sham group, MCAO group and $\mathrm{MCAO}+\mathrm{GHB}$ treated group ( $\mathrm{n}=4$ rats for each group), the rats were sacrificed after the rats received saline or GHB therapy for 5 weeks. All rats in each group were sacrificed with an overdose of $10 \%$ chloral hydrate $(400 \mathrm{mg} / \mathrm{kg})$ and then were killed by cervical dislocation (21). The mice were considered to be dead once they were without breathing and heartbeat. Then, brains were homogenized in the lysis buffer, and proteins were separated in a $4-15 \%$ gradient SDS-polyacrylamide gel and transferred to a polyvinylidene difluoride membrane. AMPK (cat. no. 5831), p-AMPK (cat. no. 50081, Thr172), p-eNOS (cat. no. 9574, Thr495) and eNOS (cat. no. 32027), $\beta$-actin (cat. no. 4970) proteins (1:1,000 dilution; Cell Signaling Technology, Inc., Boston, MA, USA) were used as an internal control controls. The blots were incubated overnight with the primary antibodies at $4^{\circ} \mathrm{C}$ in TBS containing $4 \%$ bovine serum albumin and $0.1 \%$ Tween-20. Secondary antibodies (goat anti-rabbit IgG, 1:5,000 dilution; Zhongshan Jinqiao Biotechnology Co., Ltd., Beijing) were diluted and incubated with the blots, and an ECL (pico) detection kit (Thermo Fisher Scientific, Inc., Waltham, MA, USA) was used for signal detection.

Immunostaining. Briefly, the brain sections were blocked with $10 \% \mathrm{FBS}$ for $1 \mathrm{~h}$ and then incubated with BrdU stain in $2 \mathrm{M} \mathrm{HCl}$ at $37^{\circ} \mathrm{C}$ for $20 \mathrm{~min}$ and rinsed in $0.1 \mathrm{M}$ borate buffer $(\mathrm{pH} 8.5)$ before blocking. For CD31, lectin and nestin and von Willebrand factor (1:50 dilution; Cell Signaling Technology, Inc.) staining, antigens were retrieved with citrate buffer $(10 \mathrm{mM}, \mathrm{pH} 6.5)$ for $20 \mathrm{~min}$ at $95^{\circ} \mathrm{C}$ before blocking at $4^{\circ} \mathrm{C}$ overnight. After washing, brain sections were incubated with the appropriate secondary antibodies for $1 \mathrm{~h}$. Brain sections were examined using a confocal microscope (Leica, Solms, Germany) and photographs were taken for further analysis.

Cell culture. PC12 cells, a rat cell line derived from phaeochromocytoma cells, were obtained from American Type Culture Collection (Manassas, VA, USA). PC12 cells were cultured in DMEM medium (HyClone; GE Healthcare Life Sciences, Logan, UT, USA) supplemented with $10 \%$ fetal bovine serum (HyClone; GE Healthcare Life Sciences) at $37^{\circ} \mathrm{C}$ in a humidified atmosphere containing $5 \% \mathrm{CO}_{2}$.

Transient transfection. Cells were transfected with small interfering RNA (siRNA) targeting AMPK (si-AMPK), or with negative control siRNA (NC; 5'-ACUAGUCGAUCU AUGUGUGAUATT-3') (Shanghai GenePharma Co., Ltd., Shanghai, China) using Lipofectamine ${ }^{\circledR} 2000$ (Invitrogen; 
Thermo Fisher Scientific, Inc.) at the indicated concentrations, according to the manufacturer's protocol. In brief, PC12 cells $\left(1 \times 10^{6}\right.$ cells/well) were seeded in a six well plate with $2 \mathrm{ml}$ RPMI-1640 medium. At the $60 \%$ confluence, the cells were pretreated with $10 \mu \mathrm{g} / \mathrm{ml}$ GHB for $2 \mathrm{~h}$. Subsequently, si-AMPK or NC was mixed with Lipofectamine ${ }^{\circledR} 2000$ at room temperature for $20 \mathrm{~min}$. Then, the mixture was added into each well at a final concentration of $20 \mathrm{nM}$ for $48 \mathrm{~h}$. Then, the cells were collected for further analysis.

Statistical analysis. Data were expressed as the means \pm SD. Statistics were performed with one-way analysis of variance with Tukey's post-hoc test for multiple comparisons. $\mathrm{P}<0.05$ was considered to indicate a statistically significant difference.

\section{Results}

The improvement of blood parameters and $C B F$ by GHB in $M C A O$ rats. The blood parameters, including $\mathrm{pO}_{2}, \mathrm{pH}$ and $\mathrm{pCO}_{2}$, were measured in rats. Our data showed that the $\mathrm{pO}_{2}$ values were stable after $\mathrm{MCAO}$, but the $\mathrm{pH}$ values decreased after $\mathrm{MCAO}$, and the $\mathrm{pCO}_{2}$ was enhanced $24 \mathrm{~h}$ post-MCAO, indicating that $\mathrm{MCAO}$ resulted in the respiratory depression (Fig. 1A). However, the 5-week-long GHB therapy enhanced $\mathrm{pO}_{2}$, reduced $\mathrm{pCO}_{2}$ and increased $\mathrm{pH}$ to the levels observed in the sham group (Fig. 1A). In addition, $\mathrm{CBF}$ in rats of the MCAO group was significantly decreased. However, after GHB treatment for 5 weeks, CBF was significantly increased, indicating that the MCAO-induced shortage of brain blood supply could be partially restored to the normal level by GHB treatment (Fig. 1B).

Cerebral infarction was relieved in rats after the GHB treatment. Next, TTC and H\&E staining were carried out to evaluate the effects of GHB on cerebral infarction. Not surprisingly, cerebral infarction was obvious in the MCAO group, but the infarction volume was decreased after GHB treatment (Fig. 2A). In addition, decreased neurological deficits, cerebral infarct volume and brain edema rate were found in the MCAO group, but GHB treatment improved these parameters (Fig. 2B-D). Notably, reduced neuron counts were identified in the MCAO group, but GHB treatment significantly restored the number of neurons (Fig. 2E), suggesting the protective role of GHB in cerebral infarction.

Angiogenesis was enhanced after GHB treatment. Vascular regeneration after ischemia can increase the perfusion of brain tissue and improve the repair of ischemic tissue. Here, we evaluated the density of blood vessels in the ischemic area by CD31-immunofluorescence staining. The decreased blood vessel density was found in the MCAO group, but GHB treatment enhanced the cerebral vascular density in the ischemic area (Fig. 3A). The BrdU/lectin staining was also carried out to evaluate the GHB effect on angiogenesis. As shown in Fig. 3B, MCAO surgery reduced the number of functional vessels, but GHB treatment enhanced the number of BrdU/lectin double-positive cells, suggesting the promotion of angiogenesis by GHB. Additionally, we applied endothel-specific marker (von willebrand factor, vWF) to analyze angiogenesis. As shown in Fig. 3C, positive vWF area was significantly decreased in the
MCAO group than that of sham group, but GHB treatment induced higher positive $\mathrm{vWF}$ area in the brains of MCAO rats.

Enhanced nestin-positive cells in the subventricular zone (SVZ) after GHB therapy. The presence of nestin, the marker of neural stem cells, was examined in the SVZ after ischemia. In the sham group, nestin-positive cells were sparse in the SVZ region. However, they were more obvious seven days after MCAO in the ischemic hemisphere (Fig. 4), indicating a spontaneous recovery. Interestingly, more numerous nestin-positive cells were identified in the SVZ region of the $\mathrm{MCAO}+\mathrm{GHB}$ group (Fig. 4), suggesting the proliferation of neural stem cells after GHB treatment.

GHB may improve the recovery of nerve function by activating AMPK/eNOS signaling. The general involvement of the AMPK signaling pathway in ischemic brain injury is well accepted (22). Compared with the sham group, AMPK activation was observed in the MCAO rats, suggesting that stress-mediated AMPK activation occurs after ischemia (Fig. 5A). Furthermore, the Western blot assay showed that GHB treatment further resulted in the activation of AMPK and eNOS, suggesting an enhanced energy supply. To explore the causal link between AMPK/eNOS phosphorylation and the beneficial effect of GHB, a specific siRNA targeting AMPK was selected. As shown in Fig. 5B, knockdown of AMPK reduced the phosphorylation of eNOS even in the presence of GHB. These data suggested that GHB may improve the recovery of nerve function by activating AMPK/eNOS signaling.

\section{Discussion}

GHB is an important component of Japanese green tea (Camellia sinensis), which is shown to reduce the size of cerebral infarcts following MCAO for $4 \mathrm{~h}$ in rats (1). In line with the previous studies, our data showed that GHB improves $\mathrm{CBF}$ and physiological variables, including $\mathrm{pH}, \mathrm{pCO}_{2}$ and $\mathrm{pO}_{2}$. Moreover, TTC and $\mathrm{H} \& \mathrm{E}$ staining indicated that cerebral infarction and neuronal death were decreased in the SVZ region after GHB treatment. These findings suggest that GHB directly protects rats from cerebral ischemia.

The SVZ is an important region for nerve regeneration in the adult mammalian brain $(23,24)$. Under normal conditions, the neurons migrate to the olfactory bulb and hippocampus from SVZ. Previous research has shown that increased nerve regeneration is identified in the circumstances of cerebral ischemia and hemorrhage (24). In the recovery process of stroke, post-ischemic neurogenesis plays an important role (25). Hence, the proliferation, migration, and differentiation of neural stem cells are the key therapeutic targets for the functional improvement after stroke (26). For the first time, we showed that GHB enhanced nestin-positive cells in the SVZ area, indicating enhanced neuron repair after ischemia. In line with the nerve regeneration and neuronal plasticity, there is a complex vascular remodeling during the recovery phase of ischemia. Angiogenesis is found in brain tissue after ischemia in both humans and rats (27). CD31-staining showed that GHB treatment increased the density of blood vessels in the ischemic area. Hence, GHB improves nerve regeneration and angiogenesis in the ischemic rat brains. 
A

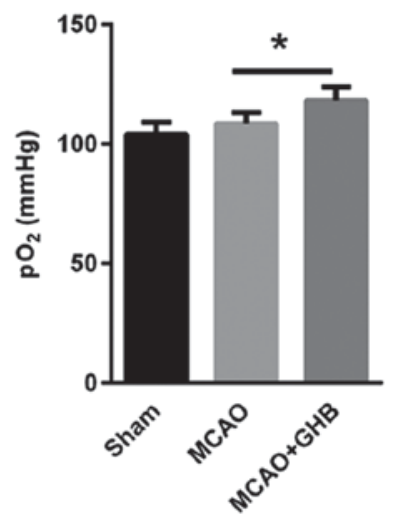

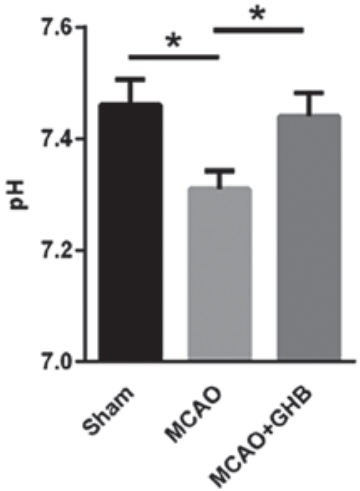

B

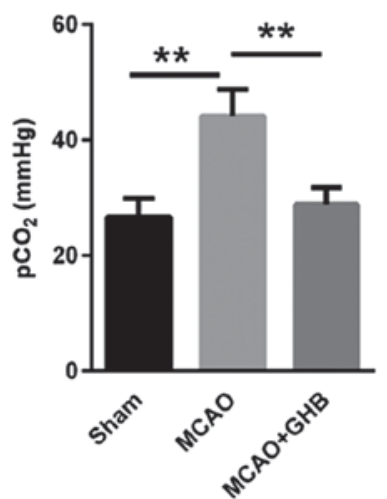

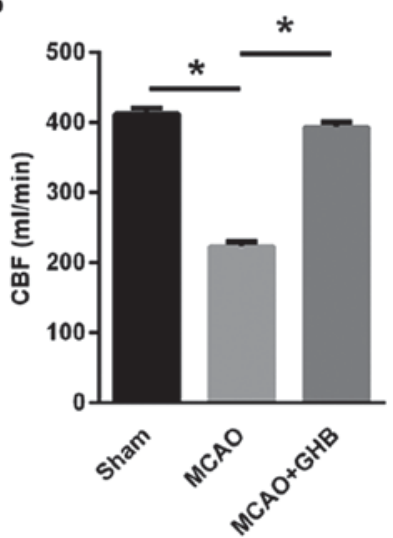

Figure 1. Improvement of blood parameters and $\mathrm{CBF}$ by $\mathrm{GHB}$ was observed in $\mathrm{MCAO}$ rats. (A) After five weeks, $\mathrm{GHB}$ therapy, increased in $\mathrm{pO}{ }_{2}$ and $\mathrm{pH}$ and a reduction in $\mathrm{pCO}_{2}$ to the levels of the sham group were observed. (B) After GHB treatment for 5 weeks, CBF was significantly increased. $\mathrm{n}=4$ biological replicates. Analysis of variance was performed. ${ }^{*} \mathrm{P}<0.05,{ }^{* * *} \mathrm{P}<0.01$ as indicated. $\mathrm{CBF}$, cerebral blood flow; GHB, hydroxybutyrate; MCAO, middle cerebral artery occlusion.

A

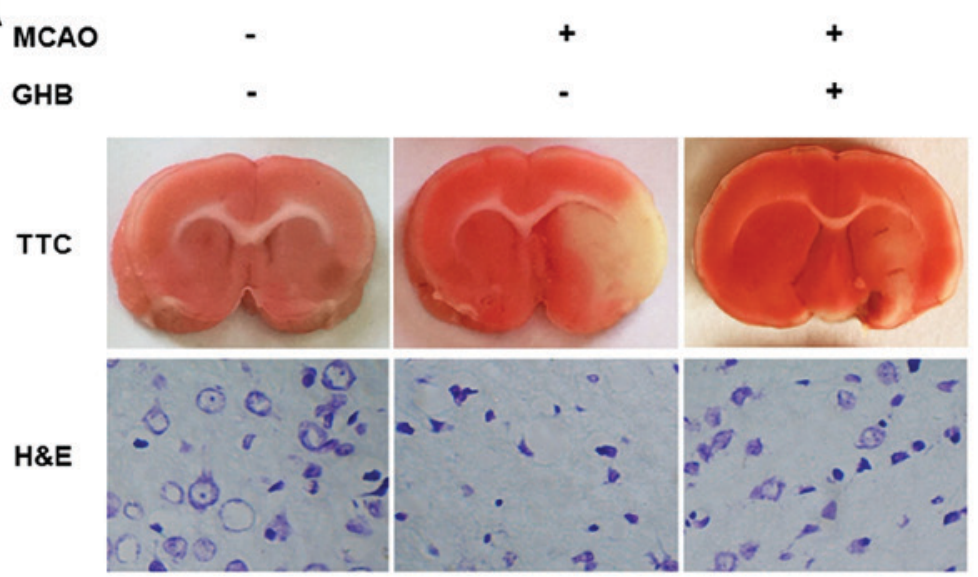

C

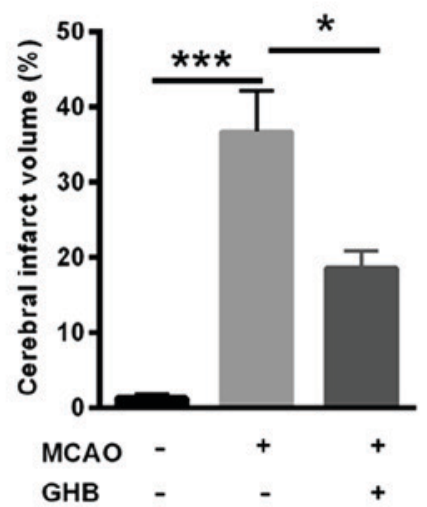

D

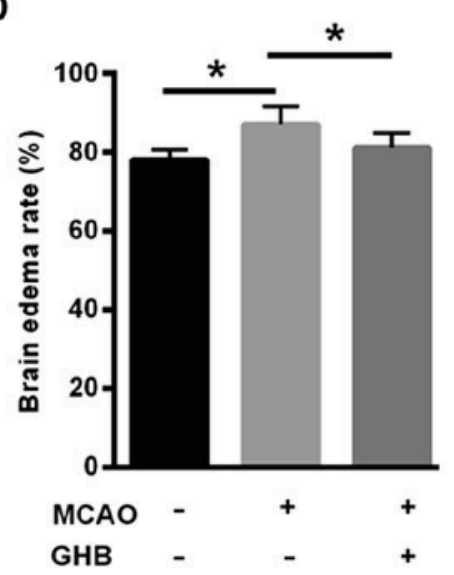

B

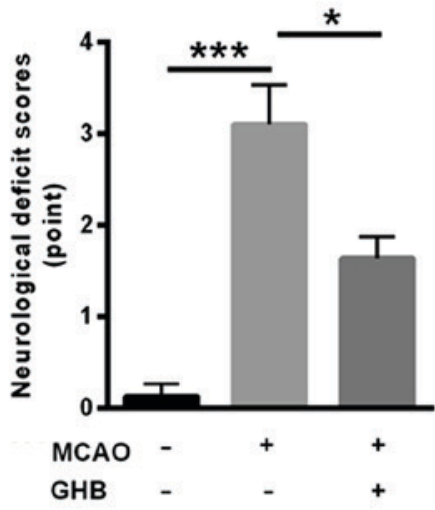

E

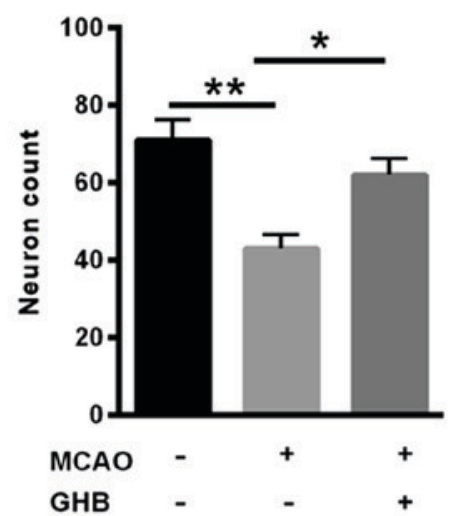

Figure 2. Cerebral infarction was relieved in rats after GHB treatment. (A) TTC staining TTC staining and H\&E staining (magnification, x100) indicated that cerebral infarction was obvious in the MCAO group, but the infarction volume was decreased after GHB treatment. (B) Decreased neurological deficits, (C) cerebral infarct volume and (D) brain edema rate were determined in all groups; GHB treatment improved these parameters. (E) Reduced neuron counts were identified in the MCAO group but GHB treatment significantly increased the number of neurons. $\mathrm{n}=4$ biological replicates. Analysis of variance was performed. ${ }^{*} \mathrm{P}<0.05,{ }^{* *} \mathrm{P}<0.01,{ }^{* * * *} \mathrm{P}<0.001$ as indicated. TTC, 2,3,5-triphenyltetrazolium; H\&E, haematoxylin and eosin; GHB, hydroxybutyrate; MCAO, middle cerebral artery occlusion.

The role of AMPK in stroke is controversial (22). For instance, several studies have shown that the acute AMPK activation is detrimental in the progression of cerebral infarction, while some other studies indicated that it is 
A

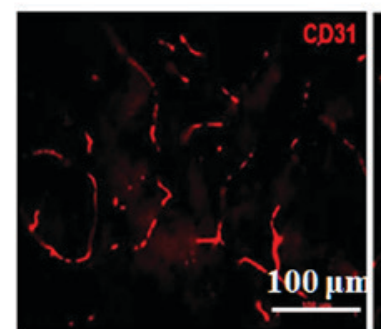

Sham

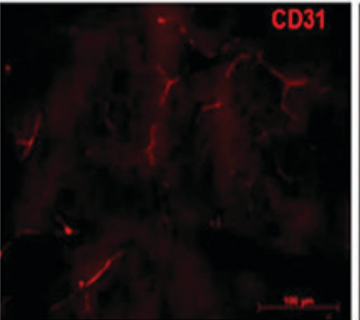

MCAO

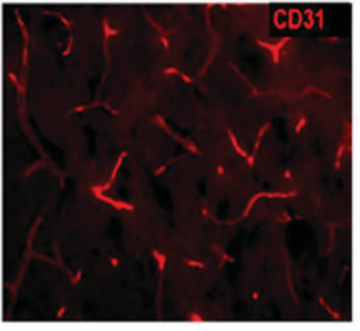

MCAO+GHB

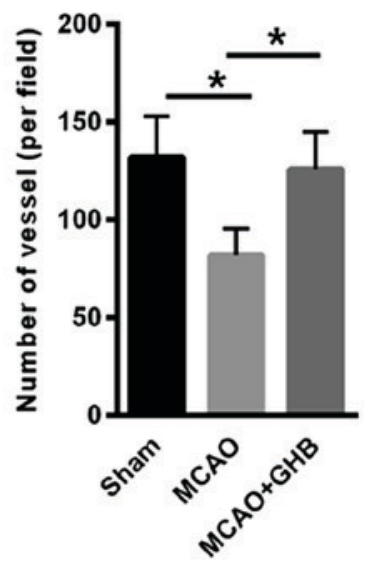

B Lectin BrdU

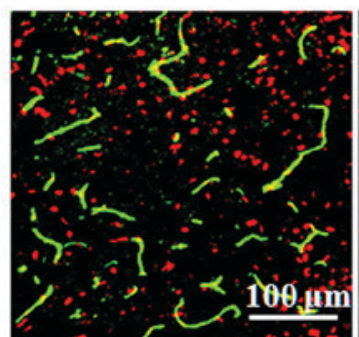

Sham

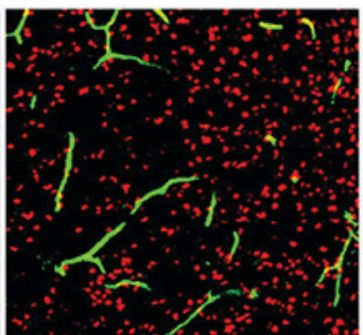

MCAO

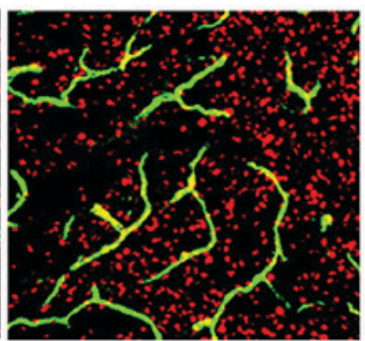

MCAO+GHB

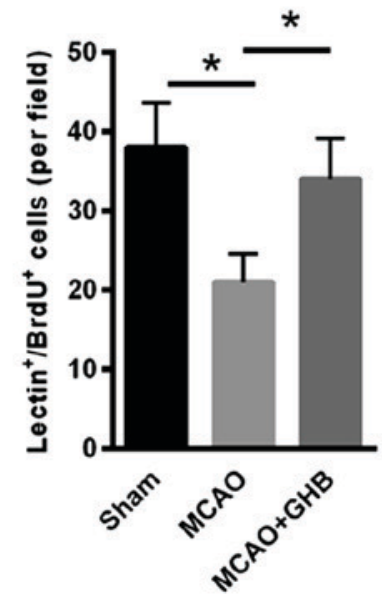

C

\section{VWF DAPI}

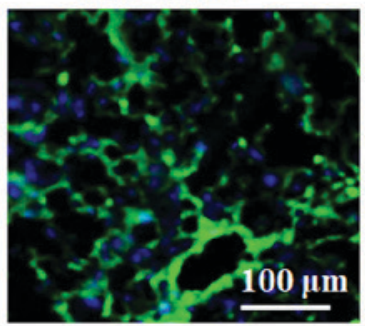

Sham

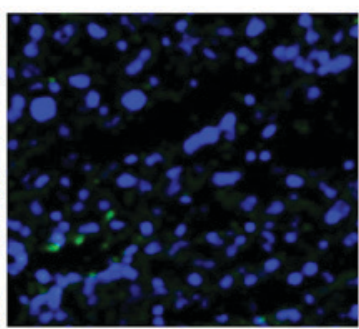

MCAO

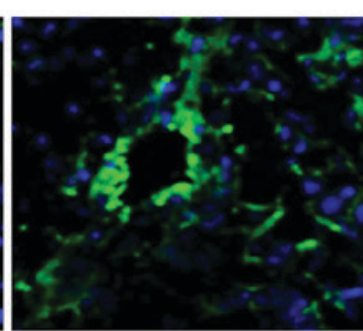

MCAO+GHB

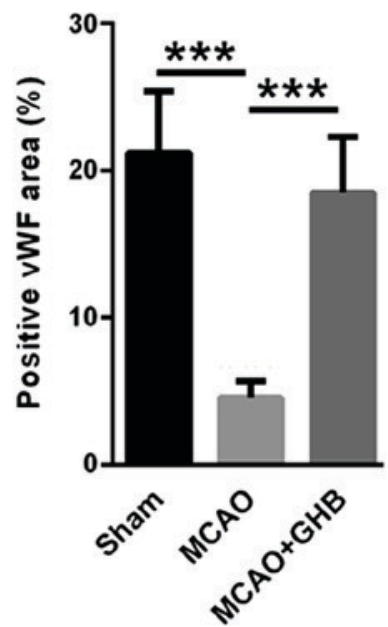

Figure 3. Immunofluorescence assay showed that GHB treatment promoted angiogenesis. (A) CD31-immunofluorescence staining indicated that GHB treatment increased the cerebral vascular density in the ischemic area. (B) GHB treatment increased the number of BrdU/lectin double-positive cells. (C) Endothel-specific marker vWF was also applied to analyze angiogenesis. $\mathrm{n}=4$ biological replicates. Analysis of variance was performed. ${ }^{*} \mathrm{P}<0.05,{ }^{* * * *} \mathrm{P}<0.001 \mathrm{as}$ indicated. $\mathrm{vWF}$, von willebrand factor; GHB, hydroxybutyrate; MCAO, middle cerebral artery occlusion.

protective (28-30). For instance, statins enhance angiogenesis partially by activating AMPK and enhancing the regeneration of vessels after cerebral ischemia (31). Moreover, AMPK could also activate eNOS in the peripheral vascular tissue $(32,33)$. In line with the previous studies, our data showed that AMPK was activated in the brains of MCAO rats. Our data indicated that the activation of AMPK and eNOS could be further enhanced by GHB, thus resulting in improved nerve vascular regeneration. Hence, we propose that activation of AMPK by GHB improves nerve regeneration, angiogenesis and brain function in the chronic recovery phase of cerebral infarction. 

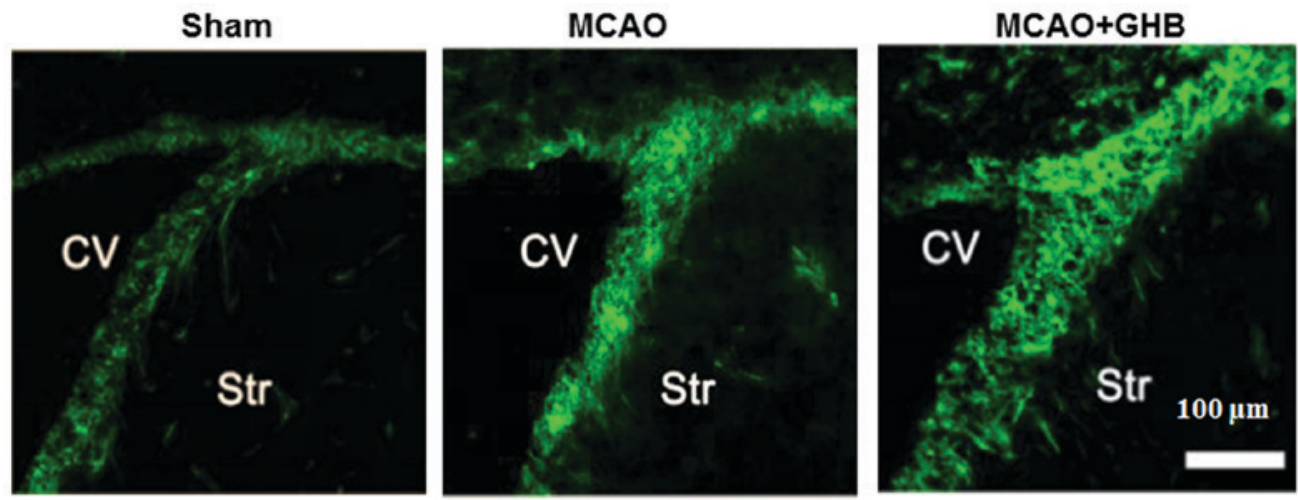

Figure 4. Immunofluorescence assay showed that GHB treatment increased the number of nestin-positive cells in the subventricular zone. CV, Corpus Striatum; Str, ventriculus lateralis cerebri; GHB, hydroxybutyrate; MCAO, middle cerebral artery occlusion.
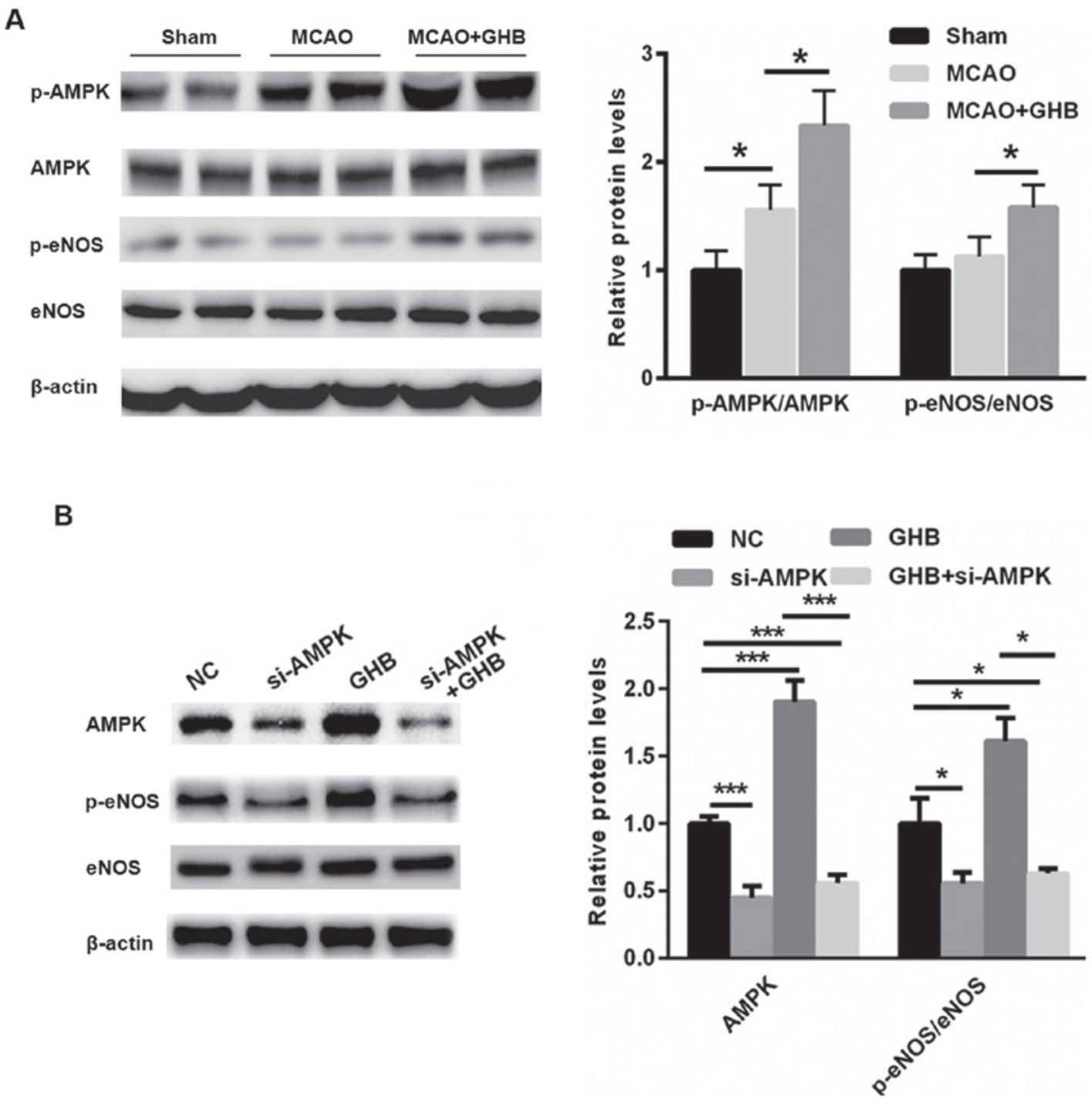

Figure 5. GHB may improve the recovery of nerve function by activating AMPK/eNOS signaling. (A) Western blot assay showed that GHB treatment resulted in the activation of AMPK and eNOS. (B) Knockdown of AMPK reduced the phosphorylation of eNOS even in the presence of GHB. $n=4$ biological replicates. Analysis of variance was performed. ${ }^{*} \mathrm{P}<0.05,{ }^{* * * *} \mathrm{P}<0.001$ as indicated. AMPK, Amp-activated protein kinase; eNOS, endothelial nitric oxide synthase; GHB, hydroxybutyrate; MCAO, middle cerebral artery occlusion.

In conclusion, for the first time, our data showed that GHB may improve the recovery of nerve function and angiogenesis by activating AMPK/eNOS signaling during the recovery period of ischemia. In the current study, it is not sufficient to demonstrate whether this mechanism underlies the recovery of nerve function following ischemia. 
In order to demonstrate this, functional assays, such as cell growth/viability in response to si-AMPK and/or GHB treatment, would need to be performed in an in vitro model of ischemic stroke.

\section{Acknowledgements}

Not applicable.

\section{Funding}

This study was supported by a grant from Zhongnan Hospital of Wuhan University Hospital (grant no. 2312087).

\section{Availability of data and materials}

The datasets used and/or analyzed during the current study are available from the corresponding author on reasonable request.

\section{Authors' contributions}

HW performed the experiments and analyzed the data. PG, XL, ZJ and XY performed the animal experiments. YW designed the experiments, analyzed the data. All authors read and approved the final manuscript.

\section{Ethics approval and consent to participate}

All experimental protocols described in this study were approved by the Ethics Review Committee for Animal Experimentation of Zhongnan Hospital of Wuhan University Hospital (no. XAERC-1002).

\section{Patient consent for publication}

Not applicable.

\section{Competing interests}

The authors declare that they have no competing interests.

\section{References}

1. Basu P, Jenkins H, Tsang K and Vakharia VN: National survey of neurosurgeons and stroke physicians on decompressive hemicraniectomy for malignant middle cerebral artery infarction. World Neurosurg 102: 320-328, 2017.

2. Friedrich B, Lobsien D, Maegerlein C, Wunderlich S, Zimmer C, Kaesmacher J and Kleine J: Distance to Thrombus in acute middle cerebral artery stroke predicts basal ganglia infarction after mechanical thrombectomy. Oncotarget 7: 85813-85818, 2016

3. Liu Y, Tang G, Li Y, Wang Y, Chen X, Gu X, Zhang Z, Wang Y1 and Yang GY: Metformin attenuates blood-brain barrier disruption in mice following middle cerebral artery occlusion. J Neuroinflammation 11: 177, 2014.

4. Bujak R, Błażejewski J, Biedermann A, Sinkiewicz W, Karasek D, Banach J and Dobosiewicz M: Severe, thromboembolic pulmonary hypertension with recurrent pulmonary embolism and right heart thrombi in a patient with past myocardial infarction, cerebral ischaemic stroke and small intestine necrosis. Kardiol Pol 69: 61-66, 2011 (In Polish).

5. De Schryver EL and Halkes PH: No role for oral anticoagulants (target INR: 2.0-3.0) after transient ischaemic attack or cerebral infarction of arterial origin; the 'European/Australasian stroke prevention in reversible ischaemia trial' (ESPRIT). Ned Tijdschr Geneeskd 152: 445-453, 2008 (In Dutch).
6. Kleindorfer D, Lindsell CJ, Brass L, Koroshetz W and Broderick JP: National US estimates of recombinant tissue plasminogen activator use: ICD-9 codes substantially underestimate. Stroke 39: 924-928, 2008

7. Wang Y, Huang Y, Xu Y, Ruan W, Wang H, Zhang Y, Saavedra JM, Zhang L, Huang $Z$ and Pang T: A Dual AMPK/Nrf2 activator reduces brain inflammation after stroke by enhancing microglia M2 polarization. Antioxid Redox Signal 28: 141-163, 2018

8. Qiu J, Wang M, Zhang J, Cai Q, Lu D, Li Y, Dong Y, Zhao T and Chen $\mathrm{H}$ : The neuroprotection of Sinomenine against ischemic stroke in mice by suppressing NLRP3 inflammasome via AMPK signaling. Int Immunopharmacol 40: 492-500, 2016.

9. Chen H, Liu H, Zou H, Chen R, Dou Y, Sheng S, Dai S, Ai J, Melson J, Kittles RA, et al: Evaluation of plasma miR-21 and miR-152 as diagnostic biomarkers for common types of human cancers. J Cancer 7: 490-499, 2016.

10. Wang LM, Wang YJ, Cui M, Luo WJ, Wang XJ, Barber PA and Chen ZY: A dietary polyphenol resveratrol acts to provide neuroprotection in recurrent stroke models by regulating AMPK and SIRT1 signaling, thereby reducing energy requirements during ischemia. Eur J Neurosci 37: 1669-1681, 2013.

11. Garcia-Prieto CF, Hernández-Nuño F, Rio DD, Ruiz-Hurtado G, Aránguez I, Ruiz-Gayo M, Somoza B and Fernández-Alfonso MS: High-fat diet induces endothelial dysfunction through a down-regulation of the endothelial AMPK-PI3K-Akt-eNOS pathway. Mol Nutr Food Res 59: 520-532, 2015.

12. Han F, Guo Y, Xu L, Hou N, Han F and Sun X: Induction of haemeoxygenase-1 directly improves endothelial function in isolated aortas from obese rats through the Ampk-Pi3k/Akt-Enos pathway. Cell Physiol Biochem 36: 1480-1490, 2015.

13. Han L, Yu Y, Sun X and Wang B: Exendin-4 directly improves endothelial dysfunction in isolated aortas from obese rats through the cAMP or AMPK-eNOS pathways. Diabetes Res Clin Pract 97: 453-460, 2012.

14. Gao B, Kilic E, Baumann CR, Hermann DM and Bassetti CL: Gamma-hydroxybutyrate accelerates functional recovery after focal cerebral ischemia. Cerebrovasc Dis 26: 413-419, 2008.

15. Kamal RM, van Noorden MS, Franzek E, Dijkstra BA, Loonen AJ and De Jong CA: The neurobiological mechanisms of gamma-hydroxybutyrate dependence and withdrawal and their clinical relevance: A review. Neuropsychobiology 73: 65-80, 2016.

16. Liechti ME, Quednow BB, Liakoni E, Dornbierer D, von Rotz R, Gachet MS, Gertsch J, Seifritz E and Bosch OG: Pharmacokinetics and pharmacodynamics of $\gamma$-hydroxybutyrate in healthy subjects. Br J Clin Pharmacol 81: 980-988, 2016.

17. Lavyne MH, Hariri RJ, Tankosic T and Babiak T: Effect of low dose gamma-butyrolactone therapy on forebrain neuronal ischemia in the unrestrained, awake rat. Neurosurgery 12: 430-434, 1983.

18. Vergoni AV, Ottani A, Botticelli AR, Zaffe D, Guano L, Loche A, Genedani S, Gessa GL and Bertolini A: Neuroprotective effect of gamma-hydroxybutyrate in transient global cerebral ischemia in the rat. Eur J Pharmacol 397: 75-84, 2000.

19. Yang C, Wang H, Zhang S, Cheng Y and Sun J: Neuroprotective effects of NKN on focal cerebral ischemia in rats. Turk Neurosurg 22: 1-6, 2012.

20. Zhang X, Chen L, Dang X, Liu J, Ito Y and Sun W: Neuroprotective effects of total steroid saponins on cerebral ischemia injuries in an animal model of focal ischemia/reperfusion. Planta Med 80: 637-644, 2014.

21. Zhang HT, Zhang P, Gao Y, Li CL, Wang HJ, Chen LC, Feng Y, Li RY, Li YL and Jiang CL: Early VEGF inhibition attenuates blood-brain barrier disruption in ischemic rat brains by regulating the expression of MMPs. Mol Med Rep 15: 57-64, 2017.

22. Ran QQ, Chen HL, Liu YL, Yu HX, Shi F and Wang MS Electroacupuncture preconditioning attenuates ischemic brain injury by activation of the adenosine monophosphate-activated protein kinase signaling pathway. Neural Regen Res 10: 1069-1075, 2015

23. Chen L, Wang X, Zhang J, Dang C, Liu G, Liang Z, Huang G, Zhao $\mathrm{W}$ and Zeng J: Tongxinluo enhances neurogenesis and angiogenesis in peri-infarct area and subventricular zone and promotes functional recovery after focal cerebral ischemic infarction in hypertensive rats. Evid Based Complement Alternat Med 2016: 8549590, 2016.

24. Liu Q, Sanai N, Jin WN, La Cava A, Van Kaer L and Shi FD: Neural stem cells sustain natural killer cells that dictate recovery from brain inflammation. Nat Neurosci 19: 243-252, 2016. 
25. Bellenchi GC, Volpicelli F, Piscopo V, Perrone-Capano C and di Porzio U: Adult neural stem cells: An endogenous tool to repair brain injury? J Neurochem 124: 159-167, 2013.

26. Christie KJ and Turnley AM: Regulation of endogenous neural stem/progenitor cells for neural repair-factors that promote neurogenesis and gliogenesis in the normal and damaged brain. Front Cell Neurosci 6: 70, 2013.

27. Yao Y, Zheng XR, Zhang SS, Wang X, Yu XH, Tan JL and Yang YJ: Transplantation of vascular endothelial growth factor-modified neural stem/progenitor cells promotes the recovery of neurological function following hypoxic-ischemic brain damage. Neural Regen Res 11: 1456-1463, 2016.

28. Ma Y, Bu J, Dang H, Sha J, Jing Y, Shan-jiang AI, Li H and Zhu Y: Inhibition of adenosine monophosphate-activated protein kinase reduces glial cell-mediated inflammation and induces the expression of $\mathrm{Cx} 43$ in astroglias after cerebral ischemia. Brain Res 1605: 1-11, 2015.

29. Nam HG, Kim W, Yoo DY, Choi JH, Won MH, Hwang IK, Jeong JH, Hwang HS and Moon SM: Chronological changes and effects of AMP-activated kinase in the hippocampal CA1 region after transient forebrain ischemia in gerbils. Neurol Res 35: 395-405, 2013.
30. Gasbarrino K, Zheng H, Hafiane A, Veinot JP, Lai C and Daskalopoulou SS: Decreased adiponectin-mediated signaling through the AdipoR2 pathway is associated with carotid plaque instability. Stroke 48: 915-924, 2017.

31. Rockberg J, Jørgensen L, Taylor B, Sobocki P and Johansson G: Risk of mortality and recurrent cardiovascular events in patients with acute coronary syndromes on high intensity statin treatment. Prev Med Rep 6: 203-209, 2017.

32. Yu JW, Deng YP, Han X, Ren GF, Cai J and Jiang GJ: Metformin improves the angiogenic functions of endothelial progenitor cells via activating AMPK/eNOS pathway in diabetic mice. Cardiovasc Diabetol 15: 88, 2016.

33. Zhang C, Liao Y, Li Q, Chen M, Zhao Q, Deng R, Wu C, Yang A Guo Z, Wang D and He X: Recombinant adiponectin ameliorates liver ischemia reperfusion injury via activating the AMPK/eNOS pathway. PLoS One 8: e66382, 2013.

This work is licensed under a Creative Commons

Attribution-NonCommercial-NoDerivatives 4.0 International (CC BY-NC-ND 4.0) License. 